\title{
Ball Valve Syndrome Caused by a Balloon-tipped Gastrostomy Tube
}

\author{
Yusaku Kajihara* \\ Department of Gastroenterology, Fuyoukai Murakami Hospital, Aomori, Japan
}

A 61-year-old man presented to the author's department with vomiting during enteral nutrition. Four months previously, the patient underwent a percutaneous endoscopic gastrostomy (PEG) due to disuse syndrome after a brain tumor surgery. In addition, a gastrostomy tube with a balloon tip had been used. The gastrostomy tube was opened to allow gastric content to flow out; however, no outflow could be observed. Emergency endoscopy revealed that the balloon tip had migrated and was stuck in the duodenal bulb (Fig. 1A), causing a gastric outlet obstruction called ball valve syndrome (BVS). Prompt deflation of the balloon was performed, and the balloon tip was returned to its proper position (Fig. 1B).

BVS was first described in 1946 by Hobbs and Cohen ${ }^{1}$ and usually refers to the gastroduodenal invagination of a large gastric polyp causing intermittent gastric outlet obstruction. BVS caused by a tip of a PEG catheter is quite rare. In this case, gastric puncture had been performed at the antrum, and the external bolster was loosened; these findings are the risk factors for BVS. Esophagogastroduodenoscopy is useful in both diagnosis and treatment of BVS. For the prevention of BVS, it is important to check whether the position of the external bolster is adequate, especially in patients using catheters with long shafts. Since BVS can lead to acute pancreatitis ${ }^{2}$ or dumping syndrome, ${ }^{3}$ prompt diagnosis and treatment are necessary.

\section{CONFLICT OF INTEREST STATEMENT}

None declared.

\section{REFERENCES}

1. Hobbs WH, Cohen SE. Gastroduodenal invagination due to a submucous lipoma of the stomach. Am J Surg 1946;71:505-18.

2. Bui HD, Dang CV. Acute pancreatitis: a complication of Foley catheter gastrostomy. J Natl Med Assoc 1986;78:779-81.

3. Shimoda M, Saraya T, Ogawa Y, Tanaka Y. Dumping syndrome due to the misplacement of the gastrostomy feeding tube. Intern Med 2015;54:2529.
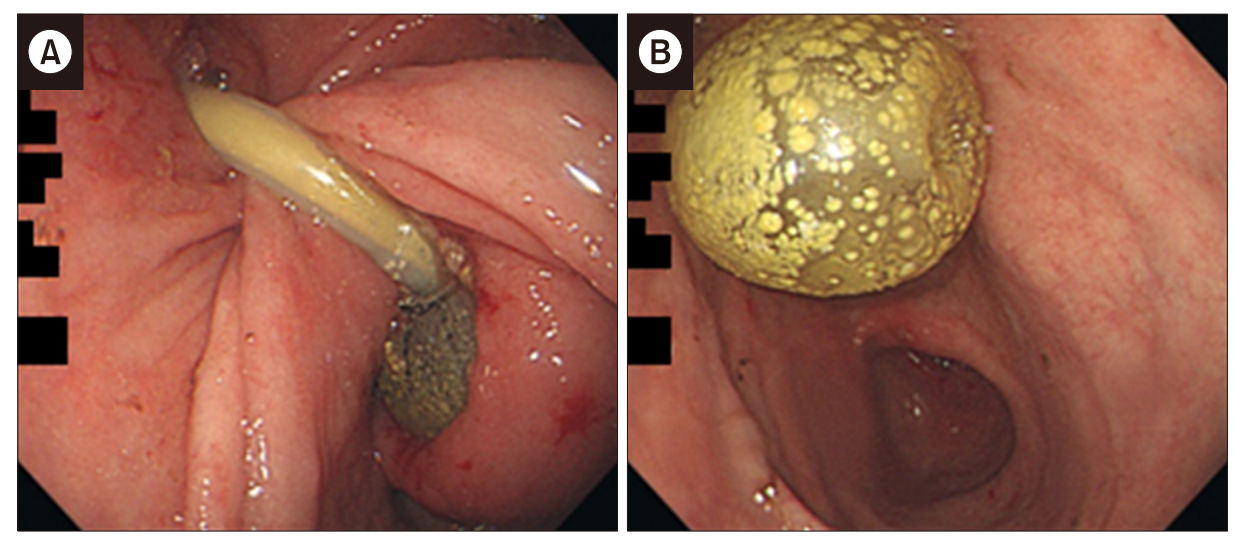

FiG. 1. (A) Endoscopic view of the gastroduodenal invagination of a balloontipped gastrostomy tube. (B) Endoscopic view of the proper position of balloon tip.

\section{Corresponding Author:}

Yusaku Kajihara

Department of Gastroenterology, Fuyoukai Murakami Hospital, 3-3-14 Hamada, Aomori 030-0843, Japan

Tel: +81-17-729-8888, Fax: +81-17-729-8887, E-mail: yukajihara-gi@umin.ac.jp

\section{Article History:}

Received September 10, 2018

Accepted September 21, 2018

This is an Open Access article distributed under the terms of the Creative Commons Attribution Non-Commercial License (http://creativecommons.org/licenses/ by-nc/4.0) which permits unrestricted non-commercial use, distribution, and reproduction in any medium, provided the original work is properly cited. 